\title{
Physiotherapy approach after lung transplantation in a critically ill COVID-19 patient: a case report
}

\author{
Veronica Rossi ${ }^{1,2}$, Serena Tammaro², Martina Santambrogio², Mariangela Retucci², Francesca Gallo ${ }^{2}$, \\ Stefania Crotti ${ }^{3}$, Marco Mantero ${ }^{2,4}$, Mario Nosotti ${ }^{4,5}$, Emilia Privitera ${ }^{1}$, Alessandro Palleschi ${ }^{4,5}$ \\ ${ }^{1}$ Health Professions Department Unit, Fondazione IRCCS Ca' Granda Ospedale Maggiore Policlinico, Milan; \\ ${ }^{2}$ Respiratory Unit and Cystic Fibrosis Adult Center, Fondazione IRCCS Ca' Granda Ospedale Maggiore Policlinico, \\ Milan; ${ }^{3}$ Department of Anesthesia, Critical Care and Emergency, Fondazione IRCCS Ca' Granda - Ospedale Maggiore \\ Policlinico, Milan; ${ }^{4}$ Department of Pathophysiology and Transplantation, University of Milan; ${ }^{5}$ Thoracic Surgery and \\ Lung Transplantation Unit, Fondazione IRCCS Ca' Granda Ospedale Maggiore Policlinico of Milan, Italy
}

\begin{abstract}
This study describes the case of an 18-years-old male affected by severe COVID-19, who was receiving bilateral lung transplantation (LT), after 71 days of mechanical ventilation and 55 days of veno-venous extracorporeal membrane oxygenation. From post-
\end{abstract}

\author{
Correspondence: Emilia Privitera, Dipartimento Professioni Sanitarie, \\ Fondazione IRCCS Ca' Granda Ospedale Maggiore Policlinico, \\ Via Sforza 28, 20122 Milan, Italy. \\ Tel. +39.338.5670909. \\ E-mail: emilia.privitera@policlinico.mi.it
}

Keywords: early rehabilitation; COVID-19; lung transplantation; functional mobilization; critical care.

Contributions: VR, ST, MR data acquisition; VR, ST, MS, FG, AP data analysis, manuscript drafting; VR, ST, MS, EP interpretation data, VR, ST, MS, AP, MM manuscript critical revision for important intellectual content, VR, ST, MS, EP, SC, MN conception and design of the work. All the authors have read and approved the final version of the manuscript and agreed to be accountable for all aspects of the work.

Conflict of interest: The authors declare that they have no competing interests, and all authors confirm accuracy.

Ethics approval and consent to participate: No ethical committee approval was required for this case report by the Department, because this article does not contain any studies with human participants or animals. Written informed consent was obtained from the patient.

Acknowledgements: Authors would like to acknowledge all the hospital staff of the Policlinico Hospital, Milan, Italy who contributed to the care of this patient.

Received for publication: 8 July 2021.

Accepted for publication: 16 September 2021.

${ }^{\circ}$ Copyright: the Author(s), 2021

Licensee PAGEPress, Italy

Monaldi Archives for Chest Disease 2022; 92:2004

doi: 10.4081/monaldi.2021.2004

This article is distributed under the terms of the Creative Commons Attribution Noncommercial License (by-nc 4.0) which permits any noncommercial use, distribution, and reproduction in any medium, provided the original author(s) and source are credited. operative day 2, early mobilization and physiotherapy treatments were performed. Weaning from mechanical ventilation, the use of non-invasive ventilation and tracheostomy management were included in the treatment. Forty-five days after LT the patient was discharged at home, showing improvements in terms of functional and respiratory parameters, quality of life and mood. While evidences about physiotherapy treatments in lung transplantation post severe COVID-19 remain limited, early approach and a multidisciplinary team may be considered key elements for functional recovery of these subjects.

\section{Introduction}

In February 2020, the first COVID-19 patient was registered in Italy and the number of infected individuals needing intensive care rapidly increased. Pneumonia associated to Severe Acute Respiratory Syndrome Coronavirus 2 (SARS-CoV-2) usually causes mild symptoms but may also lead to severe hypoxemia, which might evolve in acute respiratory distress syndrome (ARDS); in these cases, patients require intensive care unit (ICU) admission [1]. Physiotherapy can certainly play a significant role in patients' management in both moderate and severe phases of the disease [2,3]. Nevertheless, the need to isolate the patients and droplets spread during physical activity make it harder for physiotherapists to carry out their job [2,4]. Moreover, the limited knowledge of the damage the virus can cause, the presence of composite clinical pictures and the prolonged stay in the ICU present new challenges to physiotherapists. In this scenario, the option of lung transplantation (LT) for subjects with irreversible pulmonary damage calls for a definition of a potential physiotherapy approach also.

This study reports the physiotherapy management on postoperative course after LT for ARDS related to COVID-19 for the first time; informed consent was obtained from the patient. In Europe physiotherapists working in ICU and in the respiratory field are in charge of both muscoloskeletal rehabilitation and respiratory care [5].

\section{Case Report}

A young 18-year-old male underwent bilateral lung transplantation at Fondazione IRCCS Ca' Granda Ospedale Maggiore 
Policlinico of Milan (Italy) after 71 days of mechanical ventilation (MV) and 55 days on veno-venous extracorporeal membrane oxygenation (VV-ECMO) spent in another hospital in Milan for irreversible ARDS related to COVID-19 infection. Due to the prolonged ICU stay, he suffered from acquired weakness (ICU-AW). Lung transplantation was successful, and the VV-ECMO support was removed the following day.

In consideration of the virus reactivation risk after transplantation in immunosuppression regimens, he was isolated for 15 days in a dedicated room out of caution; all nursing and medical staff wore the same protections used for active COVID-19 patients.

On post-operative day (POD) 1, sedation was reduced and the patient was evaluated for the first time by the physiotherapy team (Figure 1). The patient was lucid, alert and collaborative (Richmond agitation sedation scale -1) [6], followed simple commands and received MV via oral endotracheal tube on pressure support ventilation mode (Figure 1). $\mathrm{PaO}_{2} / \mathrm{FiO}_{2}$ was 248 . At muscular assessment of the limbs, carried out using the Medical
Research Council sum score [7], he obtained a total score of 10/60 (Table 1). Spontaneous cough was not effective. Interventions were planned for 7/7 days, twice a day; in addition, both psychiatric and psychological interventions were considered.

On POD 2, the physiotherapy treatment involved early mobilization; patient positioning foresaw his turning from side to side on the bed and sitting out of bed while under mechanical ventilatory assistance. Assisted airway clearance strategies and cough function training were performed as well. After a 10-hour trial in spontaneous breathing, the patient was extubated and the physiotherapy team supported the weaning process, assessing inspiratory and peripheral muscles strength and evaluating respiratory reserve and hemodynamic response during patient positioning and early mobilization. Non-invasive ventilation (NIV) was employed to assist the patient after extubation and ensure lung expansion, adequate lung volumes and gas exchanges. On POD 4, after fever onset, the treatment was interrupted, the patient was reintubated and underwent 12-hour pronation cycle. The rapid resolution of the septic phase allowed the

Table 1. Functional, respiratory and quality of life related outcomes at different time points.

\begin{tabular}{|c|c|c|c|c|c|}
\hline & POD 2 & ICU discharge & Hospital discharge & 1 month follow up & 3 months follow up \\
\hline 6MWD (m) & NA & NA & 210 & 320 & 430 \\
\hline 1m-STST (reps) & NA & NA & 15 & 25 & 43 \\
\hline mMRC-SS score & 10 & 16 & 40 & 44 & 56 \\
\hline Ankle dorsiflexion L & 0 & 0 & 0 & 2 & 3 \\
\hline Ankle dorsiflexion $\mathrm{R}$ & 0 & 0 & 8 & 2 & 3 \\
\hline Knee extension L & 1 & 2 & 2 & 5 & 5 \\
\hline Knee extension $\mathrm{R}$ & 1 & 2 & 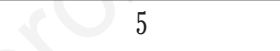 & 5 & 5 \\
\hline Hip flexion L & 2 & 2 & $8 a^{2}$ & 5 & 5 \\
\hline Hip flexion $\mathrm{R}$ & 2 & 2 & 5 & 5 & 5 \\
\hline Wrist extension L & 1 & 2 & 5 & 5 & 5 \\
\hline Wrist flexion R & 1 & 2 & 5 & 5 & 5 \\
\hline Elbow flexion L & 1 & 2 & 5 & 5 & 5 \\
\hline Elbow flexion $\mathrm{R}$ & 1 & 2 & 5 & 5 & 5 \\
\hline Shoulder abduction L & 1 & 2 & 5 & 5 & 5 \\
\hline Shoulder abduction $\mathrm{R}$ & 1 & 2 & 5 & 5 & 5 \\
\hline $\operatorname{MIP}\left(\mathrm{cm} \mathrm{H}_{2} \mathrm{O}\right)$ & NA & -35 & -65 & -75 & -120 \\
\hline $\operatorname{MEP}\left(\mathrm{cm} \mathrm{H}_{2} \mathrm{O}\right)$ & NA & NA & 60 & 72 & 125 \\
\hline FVC (L) & NA & NA & 2,17 & 2,86 & 3,2 \\
\hline FVC (\%) & NA & NA & 40 & 54 & 61 \\
\hline FEV1 (L) & NA & NA & 1,91 & 2,58 & 3,00 \\
\hline FEV1 (\%) & NA & $\mathrm{NA}$ & 43 & 58 & 68 \\
\hline PEF (L) & NA & NA & 3,45 & 4,03 & 4,39 \\
\hline PEF (\%) & NA & NA & 34 & 40 & 44 \\
\hline SVC (L) & NA & NA & 1,99 & 2,77 & 3,25 \\
\hline SVC (\%) & NA & NA & 36 & 53 & 62 \\
\hline $\mathrm{P} / \mathrm{F}$ & NA & 324 & 436 & 547 & NA \\
\hline \multirow[t]{2}{*}{ Barthel } & 25 & 85 & 100 & 100 & 100 \\
\hline & & ter surgery & 3 months after surger & 9 months afte & urgery \\
\hline
\end{tabular}

\begin{tabular}{llccc} 
SF-36, MCS & 39 & 55 & 51 \\
SF-36, PCS & 28 & 50 & 55 \\
\hline HAM-D & 18 & 6 & 0 \\
\hline
\end{tabular}

1m-STST, 1 minute sit-to-stand test, 6MWD, 6-minutes walking distance; 6MWT, 6-minutes walking test; FEV1, forced expiratory volume in the 1st second; FVC, forced vital capacity; HAM-D, Hamilton Rating Scale for Depression; ICU, intensive care unit; L, left; MCS, mental component summary; MIP, maximal inspiratory pressure; MEP, maximal expiratory pressure; MRC-SS, Medical Research Council Sum Score; PCS, physical component summary; PEF, peak expiratory flow; POD, post-operative day; R, right; SF-36, 36-Item Short Form Health Survey; SVC, slow vital capacity. 
promptly resumption of the rehabilitation program. On POD 8, surgical tracheostomy was performed (Figure 1). Swallowing was assessed and transtracheal open ventilation (TOV) was applied to facilitate patient phonation. Moreover, a home ventilator was used in invasive mode to allow early patient ambulation and movement outside his room. On POD 15, patient was progressively weaned from tracheostomy, maintaining nocturnal non-invasive ventilation (NIV) and daytime high flow nasal cannula (Figure 1).

Due to the improvement of the patient's respiratory conditions, the resolution of the neuromuscular deficit was prioritized. The patient was progressively verticalized, requiring the assistance of 2 ICU staff members.

The patient was transferred to the ward on POD 25; the functional independence in ADL measured according to the Barthel Index was 25/100 (Table 1) [8]. He underwent supervised endurance training every day with a cycloergometer and ambulated with a progressive reduction of support and training of postural changes. On POD 42, weaning from night-time NIV was performed after negative night oximetry during spontaneous breathing. Active mobilization, muscle strengthening exercises, balance training, progressive re-training of postural steps and recovery in functional activities with a particular focus on independence in ADL and walking outdoors without support were performed. The bilateral deficit of common fibular nerves was observed and studied by electromyography; to facilitate standing, walking, and stair climbing, the implantation of bilateral flexible dorsi-assist joints was prescribed.

The patient and his family were also supported with psychological interventions; the family was assisted both during the pre-
LT hospitalization period to facilitate their processing of the traumatic experience, and after the LT, as the patient initially needed to reconstruct crucial elements of his clinical history. Numerous episodes of anxiety have been observed, mainly related to the weaning from respiratory support; the patient also reported considerable fear in autonomous-breathing intervals.

Forty-five days after LT the patient was discharged at home to follow endurance training, strength muscle and walking programmes; follow-up twice a week at the hospital was planned. At discharge, pulmonary function tests and muscle strength tests were administered (Table 1). The walking distance at 6 minutes walking test (6MWT) at room air was 210 meters, whereas the repetitions at 1-minute sit to stand test (1m-STST) were 15 (Table 1) $[9,10]$.

At 1 month and 3-months after discharge, exercise capacity increased and spirometric values, quality of life parameters and patient's anxiety and depression improved (Table 1). A residual deficit of ankle dorsiflexion was observed, and dynamic-walk orthosis was still required (Table 1).

\section{Discussion}

This study reports for the first time the physiotherapy treatment administered to a patient who underwent bilateral LT for ARDS related to SARS-CoV-2. This case shows the feasibility and the advantages of incorporating physiotherapy for respiratory patients in this challenging scenario. In our hospital, the physiotherapy care of patients post LT is assured 7/7 days, twice a day, by

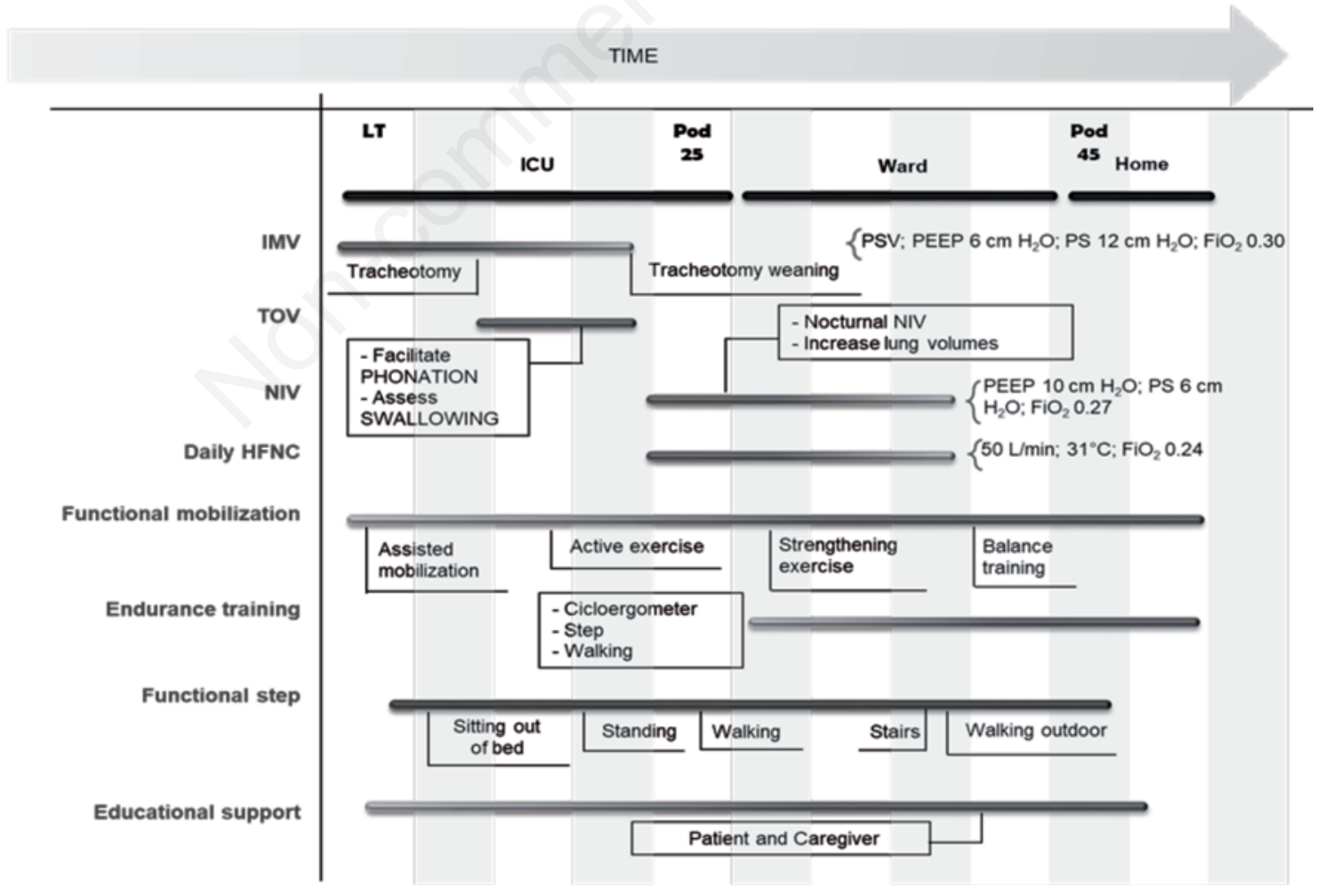

Figure 1. Timeline of physiotherapy treatments. Timeline of the hospitalization period, the interventions performed by physiotherapists and the functional steps achieved by the patient. ICU, intensive care unit; IMV, invasive mechanical ventilation; HFNC, high-flow nasal cannula; LT, lung transplantation; NIV, non-invasive mechanical ventilation; PEEP, positive end-expiratory pressure; POD, post-operative day; PS, pressure support; PSV, pressure support ventilation; TOV, transtracheal open ventilation. 
a group of 5 physiotherapists who have a postgraduate degree in cardio-pulmonary and critical care physiotherapy, supervised by a senior physiotherapist with 20-years' experience in post-transplantation care. As this patient had a new and rare condition and no treatment guidelines were available, physiotherapists planned his treatment referring to the current practice in post-transplantation and the long-term experience built in the last decade by the Lung Transplant Unit members. The case and the clinical choices were discussed by the multidisciplinary team during a daily briefing. The treatment and its progression were defined through the combined efforts of the multidisciplinary team, made of physicians, physiotherapists, psychologists, nurses, together with the patient and his caregivers. Despite the absence of formal studies on the topic, early mobilization, non-invasive ventilatory support and airways clearance seemed to be helpful to the LT population with muscle dysfunction [11]. In the given case, pre-transplantation immobilization and mechanical ventilation periods were long, resulting in dramatic ICU-AW. Compared to usual post-transplantation subjects, the treatment of this patient presented distinctive features linked to the treatment of COVID-19 infection. The need for isolation required a dedicate path of assistance due to the risk of contagion; in the early post-operative period, it was not allowed to let relatives assist the patient. The absence of support in such compromised framework, especially for a young patient, resulted in a difficult start for his daily post-operative interactions with the staff. The psychological state was certainly affected by both the long preoperative ICU stay and the poor preparation for transplantation due to the acute and rapid onset of the disease [12]. The patient, who was healthy before his COVID-19 infection, had to go through great psychological distress in having to face LT and the later acquired disability due to prolonged ICU stay. For this reason, he was supported with psychological interventions and efforts were made to help him regain his independence in ADL. In this context, the large experience of our team in the management of LT patients ensured the correct timing of physiotherapeutic interventions, such as the use of TOV to facilitate patient phonation, and the use of a home ventilator in invasive mode to allow early patient ambulation outside his room. Furthermore, the possibility of performing LT in the clinical course of selected ICU COVID-19 patients suggests careful monitoring of this category of subjects. The combined efforts of the multidisciplinary team were crucial in the patient's respiratory and functional recovery.

\section{Conclusions}

Physiotherapy after lung transplantation for ARDS related to COVID-19, was challenging compared to the usual approach. In the present report, we describe for the first time the physiotherapy management of this rare and severe condition. Further studies are needed to address the efficacy of physiotherapy in this new patient population.

\section{References}

1. Grasselli G, Greco M, Zanella A, et al. Risk factors associated with mortality among patients with COVID-19 in Intensive care units in Lombardy, Italy. JAMA Intern Med 2020;180:1345-55.

2. Lazzeri M, Lanza A, Bellini R, et al. Respiratory physiotherapy in patients with COVID-19 infection in acute setting: a Position Paper of the Italian Association of Respiratory Physiotherapists (ARIR). Monaldi Arch Chest Dis 2020;90:1285.

3. Thomas P, Baldwin C, Bissett B, et al. Physiotherapy management for COVID-19 in the acute hospital setting: clinical practice recommendations. J Physiother 2020;66:73-82.

4. World Health Organization. Clinical management of severe acute respiratory infection (SARI) when COVID-19 disease is suspected: interim guidance, 13 March 2020. Available from: https://appswhoint/iris/handle/10665/331446

5. Gosselink R, Bott J, Johnson M, et al. Physiotherapy for adult patients with critical illness: recommendations of the European Respiratory Society and European Society of Intensive Care Medicine Task Force on Physiotherapy for Critically Ill Patients. Intensive Care Med 2008;34:1188-99.

6. Sessler CN, Gosnell MS, Grap MJ, et al. The Richmond agitation-sedation scale: validity and reliability in adult intensive care unit patients. Am J Respir Crit Care Med 2002;166:1338-44.

7. Stevens RD, Marshall SA, Cornblath DR, et al. A framework for diagnosing and classifying intensive care unit-acquired weakness. Crit Care Med 2009;37:S299-308.

8. Mahoney FI, Barthel DW. Functional evaluation: The Barthel index. Md State Med J 1965;14:61-5.

9. Bohannon RW, Crouch R. 1-Minute Sit-to-Stand Test: systematic review of procedures, performance, and clinimetric properties. J Cardiopulm Rehabil Prev 2019;39:2-8.

10. Holland AE, Spruit MA, Troosters T, et al. An official European Respiratory Society/American Thoracic Society technical standard: field walking tests in chronic respiratory disease. Eur Respir J 2014;44:1428-46.

11. Langer D. Rehabilitation in patients before and after lung transplantation. Respiration 2015;89:353-62.

12. Orsini A, Corsi M, Santangelo A, et al. Challenges and management of neurological and psychiatric manifestations in SARSCoV-2 (COVID-19) patients. Neurol Sci 2020;41:2353-66. 